This item was submitted to Loughborough's Institutional Repository (https://dspace.lboro.ac.uk/) by the author and is made available under the following Creative Commons Licence conditions.

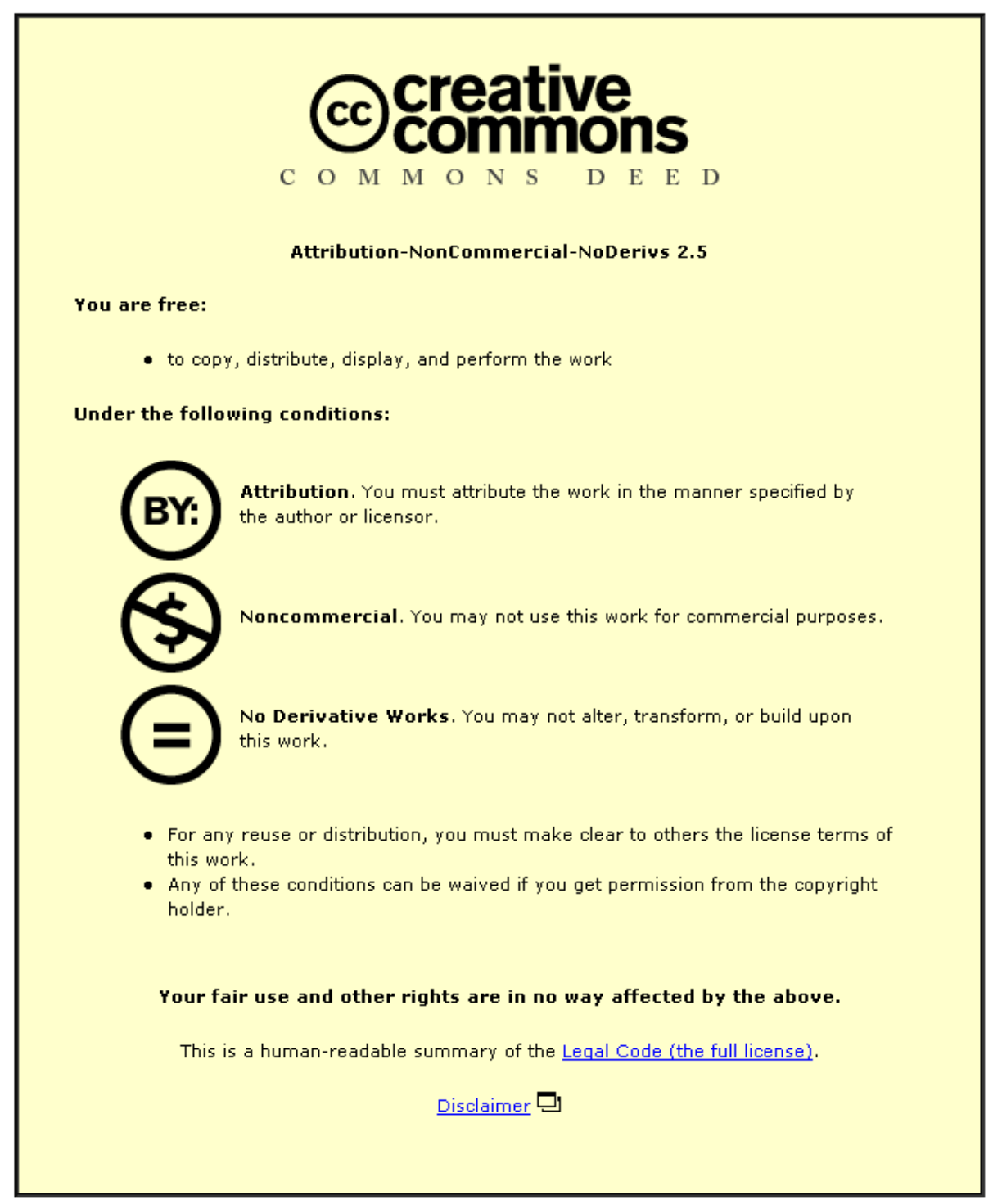

For the full text of this licence, please go to: http://creativecommons.org/licenses/by-nc-nd/2.5/ 


\title{
Dealing with Self-management of Chronic Illness at Work: Predictors for Self-disclosure
}

\author{
*Dr F. Munir, Dr S. Leka, Professor A. Griffiths
}

Published in: Social Science and Medicine, 2005, 60; 1397-1407

\author{
Institute of Work, Health \& Organisations, \\ University of Nottingham, \\ 8 William Lee Buildings \\ Nottingham Science \& Technology Park, \\ University Boulevard \\ Nottingham NG7 2RQ
}

*Corresponding author. Address as above. Email: fehmidah.munir@nottingham.ac.uk Tel: 0115846 6639. Fax: 01158466749 


\title{
Dealing with Self-management of Chronic Illness at Work: Predictors for Self-disclosure
}

\begin{abstract}
This paper explores the role of self-management of chronic illness at work, as predictors for self-disclosure. The study reports findings from a survey sent to all staff at a university, of which 610 employees reported managing a chronic illness: arthritis, musculoskeletal pain, diabetes, asthma, migraine, heart disease, irritable bowel syndrome and depression. The study found that discrete self-management factors predicted different levels of disclosure: partial self-disclosure (employees informing line managers about the presence of a chronic illness) and full self-disclosure (employees informing line managers how that chronic illness affected them at work). For partial disclosure, a greater reported experience of chronic illness by employees was positively associated with self-disclosure. For full-disclosure, employees were more likely to report disclosure to line managers if they had already disclosed to colleagues, and if they perceived receiving support from their line managers in relation to their chronic illness as important. Except for academics who were least likely to disclose, occupational groups did not emerge as significant predictors for either partial or full disclosure. Except for diabetes, chronic illness itself was not a significant predictor or barrier to self-disclosure. Our findings suggest that chronically ill employees adopt a disclosure strategy specifically related to different self-management needs of chronic illness at work.
\end{abstract}

Word count for abstract: 207

Word count for main text and references: 7498

Key words: chronic illness, self-management, disclosure, work, university 


\section{Introduction}

The impact of chronic illnesses on labour force participation has received much attention in recent years. Part of this can be attributed to the 1992 World Health Organization report in which chronic illnesses are recognised as one of the most expensive health problems in modern industrialised countries (WHO, 1992). At least a third of the workforce by the age of fifty, is likely to be managing a chronic illness, usually a cardiovascular or musculoskeletal disorder (Illmarinen, 2001). In this respect, much of the attention has been given to the workrelated causes of chronic illness (e.g. back pain), the impact of chronic illness on work productivity or to work adjustments provided (Baanders, Andries, Rijken \& Dekker, 2001; Burton, Conti, Chen, Schultz \& Edington, 1999; Gulick, Yam \& Touw, 1989; Gulick, 1992; Kessler, Greenberg, Mickelson \& Wang, 2001; Lerner, Amick, Lee, Rooney, Roger, Chang et al., 2000; Lerner, Amick, Malspeis \& Rogers, 2003; Roessler \& Rumrill, 1998). Less attention has been paid to the management of chronic illness from the employees' perspective. Self-management includes recognising and responding to symptoms, managing acute episodes, use of medication, managing relations and obtaining support from significant others, and is influenced by contextual factors such as social networks, family support, health care providers, and the physical environment (Clark, Becker, Janz, Lorig, Rakowski \& Anderson, 1991; Gallant, 2003). Whilst a significant minority of those managing a chronic illness are likely to be working, there is little evidence as to how work impacts on selfmanagement.

It is suggested that the work context may have received limited attention from researchers, due to issues associated with disclosure. Self-disclosure was defined by Collins \& Miller (1994) as the 'act of revealing personal information about oneself to another' (p.457). As a large number of chronic illnesses are hidden and not perceptible to others, receiving appropriate support from line managers and colleagues requires their knowledge and understanding of an employee's illness. Unless employees choose to inform significant 
others at work as to the nature of their illness, such support may be lacking. However, the choice to disclose a chronic illness is complex, and influenced by a host of factors such as stigma, type and severity of illness, and access to support (Greene, 2000; Vickers, 1997). There are perceived risks to the employee in disclosing a chronic illness. These include potential rejection, discrimination, loss of social support and loss of employment. Conversely, the benefits to disclosure may include access to practical and social support, social integration (e.g. Dilorio, Hennessy \& Manteuffel, 1996), ensuring as far as possible that the illness does not affect task effectiveness or performance, and re-affirming professional and work identity (Adams, Pill \& Jones, 1997; Beatty, 2001). There is currently a lack of research on self-disclosure of chronic illness at work. The purpose of the present study was to explore the self-management of chronic illness as predictors for self-disclosure of illness to line managers.

\section{Self-disclosure at work}

The extent to which an organisation is perceived to be supportive plays an extremely important role in disclosure. The existence of workplace policies and practices in supporting employees with disabilities or with an illness, such as policies on workplace diversity and equal opportunities, can positively influence self-disclosure. In addition to supportive workplace policies, other factors associated with the self-management of illness have been proposed to influence self-disclosure.

For example, self-disclosure may be necessary for the effective management of illness regimens at work (Beatty, 2001; Clark, Becker, Janz, Lorig, Rakowski, \& Anderson, 1991). In certain situations, the management of chronic illness may involve illness-specific (e.g. measuring blood glucose for diabetes) or regimen specific (e.g. keeping illness under control) activities that would be difficult to conceal. Some illnesses may necessitate regular visits to 
specialists or hospitals for treatment. Frequent workplace absences require an explanation and may precipitate disclosure, as would the use of medication or special equipment (Beatty, 2001; Clark, Becker et al., 1991). Self-disclosure may also enable employers to provide for example, tangible support that directly or indirectly facilitates self-management. Thus, an important influential factor of self-disclosure may be associated with accessing practical support. Evidence suggests that where employees do disclose, appropriate and facilitative changes in their work arrangements are more likely to occur. These include reduced working hours, decreased work pace, reduced workload, or adjustments to the physical work environment (Baanders, Andries, Rijken, \& Dekker, 2001; Daly \& Bound, 1996; Lerner, Amick, Malspeis, \& Rogers, 2000). However, not all self-disclosures result in receiving practical support (Daly \& Bound, 1996) and, equally, not all self-disclosures are related to seeking work adjustments. It is therefore not clear to what extent work adjustments alone are an influential factor for self-disclosure.

Other factors influencing the disclosure of chronic illness at work include social support. Social support is considered an important aspect of psychological adjustment for many individuals managing a chronic illness (Kalichman, DiMarco, Austin, Luke, \& DiFonzo, 2003; Petrak, Doyle, Smith, Skinner, \& Hedge, 2001; Vickers, 1997). It can buffer the stress associated with living with a chronic illness and can promote a sense of emotional well-being. Evidence from the health literature on social support suggests that in the case of chronic illness self-management, illness-specific or regimen-specific support has a stronger influence on self-management behaviour than more global types of support (Aalto, Uutela \& Aro, 1997; Gallant, 2003). Given what is known about social support in this context, we can hypothesize that there may be a similar occurrence for disclosing a chronic illness at work. Disclosing an illness may elicit social support from colleagues and line managers by increasing their understanding of the effects of the illness. Further, prior disclosures may decrease fear of disclosure and increase the practice and confidence of disclosing. For example, studies have shown that self-disclosure of personal information to family members, friends or partners, 
increases the likelihood of self-disclosure in other contexts such as the workplace (Griffith \& Hebl, 2002; Lewis, 1984). Thus, the willingness to disclose a chronic illness to line management is perhaps more likely if disclosure has already occurred elsewhere, such as with colleagues.

Although disclosure may confer benefits, there are also less positive factors to take into account. Many chronic illnesses are perceived to be stigmatising. For example, epilepsy, infertility, irritable bowel syndrome, psychological problems and HIV/AIDS are the most widely recognised stigmatising health conditions (Cline \& McKenzie, 2000; Corrigan \& Watson, 2002; Greil, 1991; Iphofen, 1990). According to Goffman (1974), stigmatised groups are viewed as 'inferior' and are discredited by groups that are considered 'normal' and in the majority. Those suffering from such stigmatising conditions are reported to not disclose their illness to their employers or colleagues, in fear of discrimination, stereotyping and prejudice (Joachim \& Acorn, 2000; Vickers, 1997). However, it is not known under what conditions the perceived risks associated with stigmatisation may be outweighed by perceived benefits of disclosure - such as use of medication or need for practical support - or vice versa. In addition to stigmatisation, organisational and team culture may also influence disclosure. From an employees' perspective, if working conditions are perceived to be negative, disclosure is less likely to take place. Studies on disclosing HIV or sexual orientation at work, suggest that self-disclosure is negatively associated with negative work attitudes, but positively associated with perceived organisational supportiveness (Griffith \& Hebl, 2002; Ragins \& Cornwell, 2001; Vivien, 2003).

Greater understanding is required as to how employees make decisions related to disclosure, if workplaces are to provide appropriate support, tackle discrimination and encourage an open culture. As outlined, several factors may be important to self-disclosure: the experience of chronic illness (e.g. use of medication or treatment), sickness absence, impact of illness on work, access to practical or social support, prior disclosure to others, possible associated 
stigma, and organisational culture. The decisions related to disclosure (e.g. if to disclose or how much to disclose) are likely to be influenced by these factors. The aim of this study was to explore the self-management of chronic illness as predictor for self-disclosure to line managers, and the extent to which self-disclosure occurs: partial disclosure (employees informing line managers about the presence of a chronic illness) and full self-disclosure (employees informing line managers how that chronic illness affected them at work). The study was conducted in a university that provided a large cohort of manual and non-manual workers, and a population diverse in terms of age, ethnic origin, disability and gender. Additionally, the organisational culture of universities is diversified across different occupational groups within the university (Gillespie, Walsh, Winefield, Dua \& Stough, 2001; Hogan, Carlson \& Dua, 2002; vanEmmerik, 2002).

\section{METHOD}

\section{Sample}

This was a cross-sectional study where all 5,000 employees from a UK university were invited to complete tailored questionnaire eliciting information on the management of chronic illness at work. The questionnaire and a letter outlining the general nature and objectives of the study was sent to all employees via the University internal mailing system. All employees were invited to volunteer for the study by completing the questionnaire. To monitor overall response rates, the questionnaire asked all employees, independent of their health status, for demographic and job-related details (age, gender, tenure and occupation). Employees managing a chronic illness were asked additional information about the nature, impact and management of their chronic illness at work. All employees were reassured that the questionnaire was confidential and individual responses would not be made known to anyone within the organisation. A 44\% response rate was achieved for completed returned questionnaires. This is an adequate response rate for mailed surveys in organisational 
research of this type (Roth \& BeVier; 1998). Out of the completed questionnaires, 734 (34\%) reported at least one chronic illness. The remaining sample did not report a chronic illness and were therefore excluded from the present analyses.

\section{Measures}

The items in the questionnaire were constructed following an analysis of the impact of published literature on chronic illness and semi-structured interviews with a representative sample of 15 employees managing a chronic illness. Employees were asked open-ended questions about the nature of their chronic illness, and the related physical, psychological and social problems that arose whilst at work. Questions covered issues of disclosure, work performance, sickness absence, and the support received or desired. Using the data from the interviews, key themes and topics were drawn and validated through content analyses. A second researcher corroborated the key themes drawn by the first author, and these were translated into context-specific questionnaire items that reflected the management of chronic illness in a work context. The questionnaire was then piloted using the interview sample. In addition, consultations were held with a group of expert stakeholders from the organisation (line managers and representatives from trade unions, the university disability committee, human resources and the occupational health unit). A draft of the questionnaire was discussed with the expert stakeholders group, and piloted with a further sample of 15 employees. Further amendments were made. The questionnaire comprised of six sections as follows: (i) employees' chronic illness, (ii) the effect of that chronic illness on work, (iii) absence attributed to that illness, (iv) disclosure and support, (v) work adjustments sought and received, and (vi) demographic details. This paper presents the findings from five of these sections as detailed below:

(i) Chronic illness was measured by asking participants to self-report on any chronic illness they currently experienced, and to indicate which primary condition (if more than one 
was listed) most affected their work. An explanation of the meaning of 'chronic illness' was provided together with examples (e.g. asthma, diabetes, back pain and arthritis). This measure was developed to be consistent with other self-report measures of chronic illness (Dewa \& Lin, 2000; Lerner, Amick et al, 2000; Kessler, Greenberg et al, 2001; Randall, Griffiths, \& Cox, 2002). Participants were asked how long they had suffered from that illness (years, months), the severity of their illness (mild, moderate or severe), frequency of symptoms associated with the illness (daily, several times a week, once a month or less than once a month). Participants were also asked if their illness had been diagnosed by a medical practitioner (e.g. their general practitioner or consultant), whether work was considered a contributing factor (i.e. the illness caused or made worse by one’s work) by their medical practitioner (yes or no), if participants used medication or special equipment (e.g. nebuliser) because of their illness whilst at work (yes or no) and if they needed time off work for treatment (yes or no).

(ii) The effect of chronic illness on work was measured by a one item question asking participants if their illness affected any aspect of their work on a daily basis. Specific examples were provided to explain this item (e.g. completing tasks on time or taking on new tasks). A dichotomous scale was used for this question (yes or no). To further measure the impact of chronic illness on work, and to assess the number of days during which participants are unproductive or unable to function at full capacity, participants were asked to rate how often they were unable to function normally at work on a five point scale (every day, several times a week, once a month, twice a year, once a year or less, or 'other'). As we were limited by questions that were asked in the survey, it was not possible to explicitly measure the effect of chronic illness across different aspects of work characteristics. However, as this study concerned a spectrum of factors associated with self-disclosure, rather than the relationship between chronic illness and specific work characteristics, occupational group was used to generally adjust for unknown work characteristics. Participants were asked two further dichotomous questions in this section, 
regarding whether they worried about their illness affecting their work (yes or no) and future career prospects (yes or no).

(iii) Absence related to chronic illness was measured by asking participants to estimate the number of times they had been absent from work over the last 12 months (spells of one day, two to four days or five days or more) because of their illness. This self-report measure is consistent with other self-report sickness absence measures (e.g. Johns, 1994a; Kirchmeyer \& Cohen, 1999). Such measures, when compared with organisational records-based absence data have a convergent validity of .62 and above (Johns, 1994a; 1994b).

(iv) Disclosure and support. Disclosure was measured by asking participants whether they had disclosed their illness to their line manager (or Head of School) and colleagues (yes or no). Participants were asked to indicate whether they had made a partial disclosure (informing their colleagues or their line manager about the presence of a chronic illness) or a full disclosure (i.e. informing their colleagues or their line manager how the illness affected them at work) (yes or no). To measure the perceived importance of workplace support in managing chronic illness (as opposed to actual support received as a result of disclosure), participants were asked to rate the importance of receiving practical support (e.g. adjustment of work arrangements) and social support (e.g. sympathy and understanding) from their line manager and their colleagues, with respect to managing their illness (very important, somewhat important or not important). For all questions in this section, participants indicated their views separately for their line manager and colleagues.

(v) Demographic information. Participants were asked to indicate their age, gender, occupation and tenure (length of employment). 


\section{Analyses}

From the 734 reported chronic illnesses, eight illness classifications emerged. Five groups were clearly identified: asthma, arthritis, irritable bowel syndrome, migraine and diabetes (Table 1). For 'depression and anxiety' participants were grouped if they reported either depression, anxiety or a combination of both. Two groups were defined using the International Classification of Diseases (ICD 10; World Health Organization, 1999). 'Musculoskeletal pain’ consisted of participants reporting pain anywhere along the musculoskeletal system (e.g. back, shoulders, neck, arms, elbows, wrist, and lower limbs). For 'heart disease', participants were included if they reported myocardial infarction, angina, heart failure, stroke and hypertension. An additional group, classified as 'Other', represented either smaller numbers of other reported chronic illnesses ( $n=46)$, or those reporting suffering from musculoskeletal pain for less than three months $(n=9)$, and from migraine or irritable bowel syndrome for less than 12 months $(\mathrm{n}=14)$. The latter was applied as a conservative measure for self-reported illnesses that might not be chronic (e.g. back strain). A further number of participants $(\mathrm{n}=55)$ did not state their illness, and were classified as 'unknown'. In order to make comparisons across the different illness groups, the latter two groups were not included in subsequent analyses (Table 1).

In order to identify significant predictors of disclosure (and thus simplify the relationships between a large number of variables), data on each aspect of chronic illness (severity, frequency of symptoms, diagnosis, whether work is a contributing factor to the chronic illness, use of medication and equipment, and time off work for treatment), work, absence and support were separately entered into a univariate logistic regression analysis against the outcome variable of disclosure (partial or full) using SPSS version 10 (SPSS, Chicago, IL). Those proven to be significant predictors at $p<0.25$ were retained (Hosmer \& Lemeshow, 1989), and for the purpose of the present analyses, items with more than two scales were dichotomised $(n=5)$, after ensuring the distribution of responses did not change significantly. 
Severity of illness was dichotomised into 'severe' (the moderate and severe scale were combined) or not severe (mild scale). Frequency of symptoms were dichotomised into 'frequent' (the categories 'every day' and 'several times a week' were combined) and 'not frequent' (the categories 'about once a month,' 'about twice a year' and 'once a year or less' were combined). For the two predictors on the importance of receiving practical support from colleagues and line managers, and the importance of receiving social support from line managers, these were dichotomised into 'important' (the scales 'somewhat important' and 'important' were combined) and 'not important' ('not important' scale). Tetrachoric correlations were then generated for all significant predictors. The correlations were retained and subjected to exploratory factor analysis with varimax rotation. Items that cross loaded were removed (two items), and the rotation was repeated until a stable structure emerged. As the variables that constituted the factors were dichotomous and coded as ' 1 ' for an event occurring, and ' 0 ' for an event not occurring, the factors were computed by the aggregation of each dichotomous score and entered into the subsequent logistic regression model as independent variables.

Using univariate logistic regression, the factors were screened for significance as predictors for partial disclosure, and separately, for full disclosure. Only those found to be significant univariate predictors of the dependent variable $(p<0.25)$, those of obvious theoretical importance (occupational group and type of chronic illness reported), and demographic variables to control for their confounding effects (length of time managing a chronic illness, age, gender and tenure) were included in analyses. Occupational groups were entered as separate variables, where a score of 1 was given if the specific occupation was present and a score of 0 given if any other occupation was present. Chronic illness groups were also entered as separate variables were a score of 1 was given if the chronic illness was present and a score of 0 given if any other chronic illness was present. Age and tenure were entered as continuous variables. Separate stepwise logistic regression analyses were carried out against each type of disclosure (partial and full). To identify the most parsimonious model of 
the data, the regression analysis was run a number of times, with the least significant variable removed from the model each time the regression was run until only a significant set $(p<.05)$ of variables remained in the model. Parameter coefficients were monitored throughout this process to ensure important variables were not removed.

\section{Results}

A total of 2172 completed questionnaires were received, representing a response rate of $44 \%$. Of these, 734 (34\%) employees reported managing a chronic illness. Table 1 shows the demographic details for participants reporting a particular chronic illness. This was compared with data obtained from the organisation's Human Resources department and indicated that the sample was representative of the population of the organisation in terms of occupational groups, age, gender and tenure.

Across the sample included in the analyses $(n=610)$, depression and anxiety were the most reported chronic illness (16.9\%), followed by asthma (13.1\%) and musculoskeletal pain (12.9\%). Across the eight chronic illness groups, 89.6\% reported their illness had been diagnosed by a medical practitioner, 26.0\% reported partially disclosing their chronic illness to their line manager (informing line manager about the presence of a chronic illness), and 24.2\% reported making a full disclosure to their line manager (informing line manager how that chronic illness affected them at work). 
Table 1: Distribution of chronic illnesses and demographic details across participants and by disclosure

\begin{tabular}{|c|c|c|c|c|}
\hline & $n$ & $\%$ & & $\begin{array}{l}\text { ial or full } \\
\text { closure } \\
\%\end{array}$ \\
\hline \multicolumn{5}{|l|}{ Chronic illnesses ( $\mathrm{n}=734)$} \\
\hline Depression \& anxiety & 124 & (16.9) & 46 & $(37.0)^{* * *}$ \\
\hline Asthma & 96 & $(13.1)$ & 45 & $(46.8)$ \\
\hline Musculoskeletal pain & 95 & (12.9) & 54 & (56.8) \\
\hline Irritable bowel syndrome & 92 & (12.5) & 34 & $(36.9) * *$ \\
\hline Arthritis & 78 & $(10.6)$ & 42 & $(53.8)$ \\
\hline Migraine & 44 & $(6.0)$ & 26 & (59.0) \\
\hline Heart disease & 44 & $(6.0)$ & 24 & $(54.5)$ \\
\hline Diabetes & 37 & $(5.0)$ & 31 & $(83.8)^{* *}$ \\
\hline Other $^{+}$ & 69 & $(9.5)$ & - & - \\
\hline Unknown $^{+}$ & 55 & $(7.5)$ & - & - \\
\hline \multicolumn{5}{|l|}{ Occupational Groupings } \\
\hline Academic & 184 & (25) & 56 & $(30.4) * * *$ \\
\hline Clerical/Administration & 175 & (24) & 106 & $(60.6)$ \\
\hline Research & 99 & (13) & 38 & (38.4) \\
\hline Technical & 97 & (13) & 51 & (52.5) \\
\hline Manual & 65 & (9) & 30 & $(46.1)$ \\
\hline Academic-related \& Management & 57 & (8) & 33 & (57.9) \\
\hline Catering \& Residential Services & 17 & (2) & 8 & $(47.0)$ \\
\hline Other & 40 & (6) & 21 & (52.0) \\
\hline \multicolumn{5}{|l|}{ Gender } \\
\hline Male & 475 & (65) & 105 & $(22.1)$ \\
\hline \multirow[t]{2}{*}{ Female } & 259 & (35) & 145 & $(55.9)^{*}$ \\
\hline & Mean & SD & & $p$ value \\
\hline Age (years) & 42.44 & (11.02) & & ns \\
\hline Tenure (years) & 8.25 & $(8.15)$ & & ns \\
\hline $\begin{array}{l}\text { Length of time managing a } \\
\text { chronic illness (years) }\end{array}$ & 11.57 & (10.17) & & ns \\
\hline
\end{tabular}

${ }^{*} p<.05, \quad{ }^{* *} p<.001, \quad{ }^{* * *} p<.0001$. Denotes respondents within the chronic illness group are significantly different from respondents within any of the other chronic illness groups.

+Respondents with 'other' and 'unknown' chronic illnesses are excluded from further analyses. 
Modelling predictors for disclosure

Data screening using univariate logistic regression identified 12 variable items significantly related to disclosure $(p<0.25)$. Variables concerning functioning at full capacity, concern for future work prospects, absence, and the importance of receiving social support from colleagues, were not significant. Factor analysis of these items revealed a stable model retaining three factors. Two items were deleted since they cross loaded on at least two factors (worry about illness affecting work and worry about future career prospects because of illness). Together, the factors explained $73 \%$ of the variance in the ten items retained (Table 2). Factor 1 contained four items that related to the importance of receiving support (explaining $28 \%$ of the variance), and included items on the importance of receiving practical support from line managers and colleagues, and social support from line managers only. The 'effect of the chronic illness on work' also loaded on this factor, associated with the importance of receiving support (Cronbach's $\alpha$.69). Factor 2 contained two items that related to colleagues knowledge about the illness, explaining 16\% of the variance (Cronbach’s $\alpha$.71). Factor 3 contained four items that related to the experience of chronic illness (explaining 22\% of the variance) and included items on the severity of chronic illness and the frequency of symptoms experienced. The use of medication or special equipment at work and time taken off work due to treatment also loaded with this factor, indicating a positive relationship between the increasing severity and frequency of symptoms, and the use of medication or needing time off work for treatment (i.e. managing their illness) (Cronbach's $\alpha$ .62). Table 3 shows the results of the screening analysis carried out before stepwise logistic regression. All three factors fulfilled the criteria for entry into stepwise logistic regression of $p<.05$ (Hosmer \& Lemeshow, 1989). 
Table 2: Rotated principal component analysis matrix for variables predicting disclosure.

\begin{tabular}{lrrr}
\hline & $\begin{array}{c}\text { Importance } \\
\text { of receiving } \\
\text { support }\end{array}$ & $\begin{array}{c}\text { Colleagues' } \\
\text { knowledge }\end{array}$ & $\begin{array}{r}\text { Experience } \\
\text { of chronic } \\
\text { illness }\end{array}$ \\
\hline Practical support from colleagues & 0.76 & -0.47 & 0.32 \\
Practical support from line manager & 0.85 & -0.18 & 0.38 \\
Social support from line manager & 0.67 & 0.42 & -0.11 \\
Effect of chronic illness on work & 0.69 & 0.01 & 0.47 \\
Colleagues' know about the illness & 0.25 & -0.83 & -0.34 \\
Colleagues know how illness affects & & & \\
the employee & -0.13 & -0.89 & 0.20 \\
Use of medication/ equipment at & & & \\
work & 0.09 & 0.02 & 0.90 \\
Time taken off work for treatment & 0.20 & 0.01 & 0.60 \\
Frequency of symptoms & 0.39 & -0.06 & 0.67 \\
Severity of illness & -0.87 & 0.09 & 0.78 \\
\hline
\end{tabular}

Table 3: Univariate predictors of the report of disclosure (significant $(p<0.25)$ predictors only)

\begin{tabular}{|c|c|c|c|c|c|c|}
\hline & \multicolumn{3}{|c|}{ Partial disclosure } & \multicolumn{3}{|c|}{ Full disclosure } \\
\hline & $\beta$ & $\begin{array}{l}\text { Odds } \\
\text { Ratio }\end{array}$ & $\begin{array}{c}\text { Confidence } \\
\text { Intervals } \\
\end{array}$ & $\beta$ & $\begin{array}{l}\text { Odds } \\
\text { Ratio }\end{array}$ & $\begin{array}{c}\text { Confidence } \\
\text { Intervals }\end{array}$ \\
\hline Importance of receiving support & 0.65 & $1.91 * * *$ & $1.48-2.48$ & 1.40 & $4.07 *$ & $2.65-6.27$ \\
\hline Colleagues’ knowledge & 0.30 & $1.34^{*}$ & $1.01-1.90$ & 1.41 & $4.11^{* *}$ & $2.68-6.31$ \\
\hline Experience of chronic illness & 0.46 & $1.59 * *$ & $1.19-2.10$ & 0.74 & $2.10^{*}$ & $1.28-3.43$ \\
\hline
\end{tabular}

\section{Predictors of partial disclosure}

Stepwise logistic regression identified a parsimonious model of the data $\left(\chi^{2}=17.24, \mathrm{df}=1\right.$; $p<.0001$ ), that contained two significant predictors of partial disclosure (reporting the presence of a chronic illness to the line manager) (Table 4). The parsimonious model accurately classified $70 \%$ of the cases, with $69 \%$ of those not reporting partial disclosure, and 71\% reporting partial disclosure accurately classified. In the parsimonious model, academics were significantly less likely to report disclosure than those in other occupational groups. By 
taking the reciprocals of the odds ratios, it can be seen that non-academics were three times more likely to report partial disclosure than academics. Those reporting diabetes were more likely to report partial disclosure (OR 1.38, CI 1.02-15.59), and those scoring high on the experience of chronic illness (e.g. reporting moderate to severe chronic illness, using medication or special equipment) were also more likely to report partial disclosure (OR 1.51, CI 1.10-2.08). The other factors were not significant in the model. Except for diabetes, the type of chronic illness reported, and potential confounding variables were not significant and did not markedly change other parameter coefficients.

Table 4 Factors explaining partial disclosure of chronic illness to line manager

\begin{tabular}{lrll}
\hline & $\beta$ & $\begin{array}{l}\text { Odds } \\
\text { Ratio }\end{array}$ & $\begin{array}{l}\text { Confidence } \\
\text { Intervals }\end{array}$ \\
\hline & & & \\
$\begin{array}{l}\text { Academic } \\
\text { Diabetes }\end{array}$ & -1.25 & $0.29^{* *}$ & $0.14-0.58$ \\
$\begin{array}{l}\text { Experience of chronic } \\
\text { illness }\end{array}$ & 0.40 & $1.99^{*}$ & $1.02-15.59$ \\
\hline${ }^{*} p<.05,{ }^{* *} p<.01$ & & & $1.10-2.08$ \\
\hline
\end{tabular}

\section{Predictors of full disclosure}

Two independent variables were found to be significant predictors of full disclosure (reporting the presence of a chronic illness, and how it affects the employee at work to the line manager $)$ in the most parsimonious model of the data $\left(\chi^{2}=16.27, \mathrm{df}=1 ; p<.0001\right)$. (Table 5). The parsimonious model accurately classified $72 \%$ of the cases, with $70 \%$ of those not reporting full disclosure, and 75\% reporting full disclosure accurately classified. In the parsimonious model, the importance of receiving support was a significant predictor in reporting full self-disclosure (OR 1.68, CI 1.03-2.73); and colleagues' knowledge of chronic illness was also significantly related to full self-disclosure to line managers. The other factors were not significant in the model. The type of chronic illness reported, and potential 
confounding variables were not significant and did not markedly change other parameter coefficients.

Table 5 Factors explaining full disclosure of chronic illness to line manager

\begin{tabular}{ccll}
\hline & $\beta$ & $\begin{array}{l}\text { Odds } \\
\text { Ratio }\end{array}$ & $\begin{array}{l}\text { Confidence } \\
\text { Intervals }\end{array}$ \\
\hline & & & \\
\hline $\begin{array}{l}\text { Importance of receiving support } \\
\text { Colleagues' knowledge }\end{array}$ & 0.52 & $1.68^{*}$ & $1.03-2.73$ \\
& 1.66 & $5.26^{* * *}$ & $2.10-13.02$ \\
\hline${ }^{*} p<.05,{ }^{* *} p<.0001$ & & & \\
\hline
\end{tabular}

\section{Discussion}

The aim of this study was to explore the role of self-management of chronic illness as predictors for self-disclosure at work. Almost half of respondents reported disclosing their chronic illness to their line manager (partial or full disclosure). The study identified different self-management factors associated with partial self-disclosure and full self-disclosure, indicating a 'need-to-know basis' disclosure strategy adopted by all chronic illness groups. This strategy suggests that chronically ill employees are likely to disclose if there is a clear need or reason for disclosure to occur, and in so doing, control the amount of information disclosed.

This study found that a greater reported experience of chronic illness is positively associated with partial self-disclosure, and supports the argument that disclosure is necessary for the effective management of illness regimens at work (Beatty, 2001). In other words, if an employee's experience of their chronic illness is increasingly frequent or severe, or requires use of medication at work or treatment, they are more likely to partially disclose their illness 
to their line manager, regardless of the nature of their chronic illness. This is further supported by the finding that with the exception of diabetes, reporting particular chronic illnesses were not significant predictors or barriers to self-disclosure. It is not clear why diabetes alone was significantly associated with partial self-disclosure. It may reflect among diabetics a greater need for self-management behaviours that is specific to their illness, which was not measured in this study. For example, compared with the aetiology of other chronic illnesses reported in the study, diabetes is more frequently associated with complications, requires stricter diet regimens, medication adherence and glucose testing (Goodall \& Halford, 1991).

Our findings do not corroborate with studies that suggest those managing stigmatised chronic illnesses, such as depression and irritable bowel syndrome, are less likely to disclose their illness (e.g. Corrigan \& Watson, 2002). As these illnesses were not related to lower disclosure rates, it is likely that the progressive and accepting culture of the organisation may have influenced positive self-disclosure behaviour. However, as a direct measure of stigma was not used in this study, it is possible that our findings do not fully capture the selfperceptions of stigma of chronically ill employees. In addition, as the study achieved a 34\% response rate for reported chronic illnesses, employees with stigmatising conditions were perhaps less likely to respond to the survey. It is also plausible that those affected by negative social values in the workplace leave the organisation and therefore their views were not included in the study.

In the full self-disclosure model, disclosing a chronic illness to colleagues at any level (i.e. partial or full) was significantly related to reporting full self-disclosure to line managers. This finding adds to the disclosure literature (e.g. Griffith \& Hebel, 2002; Lewis, 1984) in that selfdisclosure to others is more likely to occur if it has already occurred elsewhere. The disclosure of chronic illness to colleagues, in this study, may have encouraged an open culture among colleagues, and thus, given the employee the self-assurance to fully disclose to line 
managers. However, it is plausible that an already open workplace culture facilitated selfdisclosure. Disclosing to colleagues may have also resulted in more favourable colleague reactions, further encouraging full disclosure. On the other hand, our findings may also suggest that if colleagues know about an employee’s chronic illness, it may become difficult for such employees to hide the illness from their line managers, thus prompting disclosure. Overall, self-disclosure to colleagues and line managers may suggest a self-management strategy in maintaining relations with 'significant others’ (Clark, Becker et al., 1991; Gallant, 2003). Such a strategy may be directly or indirectly related to self-efficacy (Clark, Becker et al., 1991; Clark \& Dodge, 1999), i.e. the employee’s confidence in his/her ability to undertake specific self-management behaviours at work, and outcome expectations (Bandura, 2004), i.e. the outcome the employee expects his/her self-management action to produce. Both selfefficacy and outcome expectations have been highlighted as main influences on successful self-management of chronic illness (Clark \& Dodge, 1999). However, at present, relatively few studies have examined the influence of self-efficacy and outcome expectations on selfmanagement in relation to contextual factors such as social networks, families, the physical environment and the workplace.

The importance of receiving both practical (e.g. work adjustments) and social support in relation to work being affected by the chronic illness, was a significant predictor of fulldisclosure to line managers by all chronic illness groups. Again, this result is consistent with the extant literature, in that an integral part of successful management of chronic illness is associated with receiving practical and social support specific to the needs of the chronically ill (Aalto, Uutela \& Aro, 1997; Gallant, 2003). This can only be achieved if the provider of the support, in this case the line manager, is in possession of the necessary information i.e. how the chronic illness affects the employee at work.

Except for academics, occupational groups did not emerge as significant predictors for either partial or full disclosure. For partial disclosure, academics were least likely to disclose a 
chronic illness to their line manager (i.e. Head of Schools). A possible explanation may lie in the organisational culture of flexible working hours for academics, making it easier for them to work from home and manage their illness without the need for disclosure at work. Alternatively, although work characteristics were not measured in this study, evidence from the literature suggests that compared with other occupations (e.g. manual workers, clerical staff, or support staff), employees are less likely to disclose their illness if they are working in professional groups such as teaching, due to the pressures of maintaining competence, reliability and credibility (Beatty, 2001), part of which can be attributed to the general stigma attached to any group at risk of being perceived as a burden to achieving the goals of the workforce (for example, part-time workers, particularly women (Brown, Caraway, Brady, Iwamasa \& Caldwell, 2002; Burström, Holand, Diderichsen \& Whitehead, 2003), those with children (Lewis \& Cooper, 1999) or those with disabilities or chronic illnesses). As the academic group did not emerge as a significant barrier to full disclosure, it is plausible that the need for support took priority over any workplace culture or attitude perceived by chronically ill employees. Thus, those with chronic illnesses are perhaps more likely to take the risk of disclosure in order to access the support they need to manage their illness, complete their job tasks and continue with their employment.

There is some evidence to suggest that chronic illnesses are associated with high rates of sickness absence (e.g. Grossi, Soares et al., 1999; Poole, Gibbons \& Calvert, 1994). In this study, absence was not a significant predictor of disclosure. As this was a self-report measure, it is possible that participants were either under-reporting absence or were attending work when feeling unwell. However, the inability to function to full capacity measure, was also not a significant predictor of disclosure. This finding must be interpreted with caution, as the organisational culture of working hours for academic and research staff is different to other organisations (as discussed earlier). For example, the flexibility to arrive late, leave early, or to work from home means that for many of these chronically ill employees, it may be 
easier to mask an episode of illness which would typically prevent an employee from attending work.

There are several limitations to this study. The $34 \%$ response rate for reported chronic illnesses may be regarded as a study limitation in terms of generalizability of findings. Because of time and length limitations, it was not possible to include all measures of illness management, such as diet and exercise. The influence of organisational culture, work characteristics and individual differences such as illness identity, coping and psychological well-being on disclosure could also not be assessed. Also, the study did not measure barriers to disclosure such as fear of rejection and poor self-esteem. This study focused on the primary reported condition most affecting work. Many chronic illnesses however, are subject to one or several other associated chronic conditions, such as depression (e.g. Sykes, Blanchard, Lackner, Keefer \& Krasner, 2003), which can further complicate self-management of such illnesses. For future studies, a model is perhaps needed which tests for these relationships in both self-management and in disclosure. Moreover, the relationship between the outcomes of disclosure and self management needs to be examined within a work context. Evidence from the health literature suggests that practical and social support, and social interactions have a direct or indirect influence on self-management outcomes (Berkman \& Glass, 2000; Kaplan \& Toshima, 1990). Finally, the study relied entirely on self-report data in identifying those with chronic illnesses, which may have either resulted in under-reporting of chronic illnesses, leading to a somewhat lower response rate, or to an over-presentation of chronic illnesses that are not medically diagnosed.

This study identified key self-management factors as predictors of self-disclosure of chronic illnesses to line managers. We implicate a strategy employed by chronically ill employees, in that such employees control the level of information disclosed, according to their selfmanagement needs. We also acknowledge that disclosure itself is a self-management strategy, and that self-disclosure may facilitate self-management. For example, in this study, 
absence and functioning at full capacity may have been positively affected as a result of selfdisclosure.

Although this study was carried out in a university setting, with some findings specific to the education sector, the implications can be generalised both in practice and in employment. First, from a health professional's perspective, although the importance of the social context within which self-management occurs is already acknowledged by both medical practitioners and health educators, a greater understanding is needed for work as a contextual factor. Second, from an employer's perspective, our findings highlight the importance of creating a supportive work environment for chronically ill employees, which may help such employees to not only effectively manage their illness at work, but also minimise the extent to which it may affect work itself. For example, by introducing work adjustment policies, making provisions for practical and social support, and encouraging disclosure. Employees who disclose are likely to benefit from work adjustments, social support, adjustments to their sickness absence records and improved overall quality of working life. However, one of the main problems in creating a responsive and supportive work environment is overcoming barriers to disclosure and attitudes towards chronic illness by employers.

Issues concerning disclosures of chronic illness in the workplace are complex but can be understood with an increased focus on the workplace experiences of chronically ill employees. Recent European and national legislation such as the UK Disability Discrimination Act 1995 and the EU Council Directive 2000/78/EC, is directing attention to promoting the participation of people with illness and disabilities in working life, both in order to prevent their social exclusion and also as a solution to the problem of shortages in the labour market. Given that such legislation and organisational policies are continually changing, and self-management interventions continually evolving, it is important to continue to empirically examine critical issues that chronically ill employees face. 


\section{REFERENCES}

Aalto, A. M., Uutela, A., \& Aro, A. R. (1997). Health related quality of life among insulindependent diabetics: Disease-related and psychosocial correlates. Patient Education \& Counseling, 30, 215-225.

Adams, S., Pill, R., \& Jones, A. (1997). Medication, chronic illness, and identity: the perspectives of people with asthma. Social Science \& Medicine, 45 (2), 189-201.

Baanders, A. N., Andries, F., Rijken, P. M., \& Dekker, J. (2001). Work adjustments among the chronically ill. International Journal of Rehabilitation Research, 24, 7-14.

Bandura, A. (1977). Self-efficacy: toward a unifying theory of behavioural change. Psychological Review, 84, 191-215.

Bandura, A. (2004). Health promotion by social cognitive means. Health Education \& Behaviour, 31 (2), 143-164,

Beatty, J. (2001). Chronic illness identity in the workplace. Academy of Management Conference, Washington, August.

Berkman, L.F., \& Glass, T. (2000). Social integration, social networks, and health. In L.F. Berkman \& I. Kawachi (Eds.), Social epidemiology (pp. 137-173). New York: Oxford University Press.

Brown, E. J., Caraway, S. J., Brady, K. L., Iwamasa, G. Y., \& Caldwell, C. T. (2002). Taking steps as women in academia: Struggles and solutions. Behavior-Therapist, 25 (10), 189-192. 
Burström, B., Holland, P., Diderichsen, F., \& Whitehead, M. (2003). Winners and losers in flexible labor markets: The fate of women with chronic illness in contrasting policy environments - Sweden and Britain. Labor Markets and health: AN International Comparison, 33 (2), 199-217.

Burton, W. N., Conti, D. J., Chen, C-Y., Schultz, A. B., Edington, D. W. (1999). The role of health risk factors and disease on worker productivity. Journal of Occupational Environmental Medicine, 41 (10), 863-877.

Clark, N. M., Becker, M. H., Janz, N. K., Lorig, K., Rakowski, W., \& Anderson, L. (1991). Self-management of chronic disease by older adults. Journal of aging \& Health, 3, 3-27.

Clark, N. M., \& Dodge, J. A. (1999). Exploring self-efficacy as a predictor of disease management. Health Education \& Behavior, 26, 72-89.

Cline, R. J. W., \& McKenzie, N. (2000). Dilemmas of disclosure in an age of HIV/AIDS: Balancing privacy and protection in the healthcare context. In S. Petrino (Eds.), Balancing the secrets of private disclosures (pp.71-82). New Jersey: Lawrence Erlbaum Associates.

Collins, N. L., \& Miller, L. C. (1994). Self-disclosure and liking: A meta-analytic review. Psychological Bulletin, 116, 457-475.

Corrigan, P. W. \& Watson, A. C. (2002). The paradox of self-stigma and mental illness. Clinical Psychology-Science \& Practice, 9, 35-53.

Daly, M. C., \& Bound, J. (1996). Worker adaptation and employer accommodation following the onset of a health impairment. Journal of Gerontology, 51B (2) S53-S60. 
Dewa, C. S., \& Lin, E. (2000). Chronic physical illness, psychiatric disorder and disability in the workplace. Social Science \& Medicine, 51, 41-50.

Dilorio, C., Hennessy, M., Manteuffel, B. (1996). Epilepsy self-management. A test of a theoretical model. Nursing Research, 45, 211-217.

Disability Discrimination Act (1995). London: Her Majesty’s Stationary Office.

Farber, B. A. (2003). Patient self-disclosure: A review of the research. Journal of Clinical Psychology, 59, 589-600.

Gallant, P. (2003). The influence of social support on chronic illness self-management: A review and directions for research. Health Education \& Behavior, 30 (2), 170-195.

Gillespie, N. A., Walsh, M., Winefield, A. H., Dua, J., \& Stough, C. (2001). Occupational stress in universities: Staff perceptions of the causes, consequences and moderators of stress. Work \& Stress, 15 (1), 53-72.

Goffman, E. (1974). Stigma,: Notes on the management of spoiled identity. New York: Jason Aronson.

Goodhall, T. A., \& Halford, W. K. (1991). Self-management of diabetes mellitus; A critical review. Health Psychology, 6, 387-398.

Greene, K. (2000). Disclosure of chronic illness varies by topic and target: The role of stigma and boundaries in willingness to disclose. In S. Petrino (Eds.), Balancing the secrets of private disclosures (pp.123-135). New Jersey: Lawrence Erlbaum Associates. 
Greil, A. L. (1991). A secret stigma: The analogy between infertility and chronic illness and disability. Advances in Medical Sociology, 2, 17-38.

Griffith, K. H., \& Hebl., M. R. (2002). The disclosure dilemma for gay may and lesbians: “Coming out” at work. Journal of Applied Psychology, 87, 1191-1199.

Grossi, G., Soares, J. F., Ängesleviä, J., \& Perski, A. (1999). Psychosocial correlates of longterm sick leave among patients with musculoskeletal pain. Pain, 80, 607-619.

Gulick, E. E. (1992). Model for predicting work performance among persons with multiple sclerosis. Nursing Research, 41 (5), 266-272.

Gulick, E. E., Yam, M, \& Touw, M. M. (1989). Work performance by persons with multiple sclerosis: conditions that impede or enable the performance of work. International Journal of Nursing Studies, 26, 301-311.

Hogan, J. M., Carlson, J. G., \& Dua, J. (2002). Stressors and stress reactions among university personnel. International Journal of Stress Management, 9 (4), 289-310.

Hosmer, D. W., \& Lemeshow, S. (1989). Applied Logistic Regression. New York: John Wiley.

Ilmarinen J. (1994) Ageing, work and health. In: J Snel \& R. Cremer (Eds.), Work and ageing: A European perspective. London: Taylor \& Francis.

Iphofen, R., (1990). Coping with a "perforated life": A case study in managing the stigma of petit mal epilepsy. Sociology, 24, (3), 447-463. 
Joachim, G., \& Acorn, S. (2000). Stigma of visible and invisible chronic conditions. Journal of Advanced Nursing, 32, 243-248.

Johns, G. (1994a). Absenteeism estimates by employers and managers: divergent perspectives and self-serving perceptions. Journal of Applied Psychology, 79, 229-239.

Johns, G. (1994b). How often were you absent? A review of self-reported absence data. Journal of Applied Psychology 79 (4), 574-591.

Kalichman, S. C., DiMarco, M., Austin, J., Luke, W., \& DiFonzo, K. (2003). Stress, socialsupport, and HIV-status disclosure to family and friends among HIV-positive men and women. Journal of Behavioral Medicine, 26 (4), 315-332.

Kaplan, R. M., \& Toshima, M. T. (1990). The functional effects of social relationships on chronic illnesses and disability. In B.R. Sarason, I.G. Sarason \& G. R. Pierce (Eds.), Social support: An interactional view. New York: John Wiley.

Kessler, R.C., Greenberg, P.E., Mickelson, K.D., \& Wang, P.S. (2001). The effects of chronic medical conditions on work loss and work cutback. Journal of Occupational and Environmental Medicine, 43, 218-225.

Kirchmeyer, C., \& Cohen, A. (1999). Different strategies for managing the work/non-work interface: A test for unique pathways to work outcomes. Work \& Stress, 13 (1), 59-73.

Lerner, D., Amick, B. C., Malspeis S., \& Rogers, W. H. (2000). A national survey of healthrelated work limitations among employed persons in the United States. Disability and Rehabilitation, 22, 225-232. 
Lerner, D., Amick, B. C., Lee, J. C., Rooney, T., Rogers, W. H., Chang, H., Berndt, E. R. (2003). Relationship of employee-reported work limitations to work productivity. Medical Care, 41 (5), 649-659.

Lewis, L. A. (1984). The coming-out process for lesbians: Integrating a stable identity. Social Work, 29, 464-469.

Lewis, S., \& Cooper, C. L. (1999). The work-family research agenda in changing contexts. Journal of Occupational Health Psychology, 4, 382-393.

Petrak, J. A., Doyle, A. M., Smith, A., Skinner, C., \& Hedge, B. (2001). Factors associated with self-disclosure of HIV serostatus to significant others. British Journal of Health Psychology, 6, 69-79.

Poole, C. J., Gibbons, D., Calvert, I. A. (1994). Sickness absence in diabetic employees at a large engineering factory. Occupational Environmental Medicine, 51, 299-301.

Ragins, B. R., \& Cornwell, J. M. (2001). Pink triangles: Antecedents and consequences of perceived workplace discrimination against gay and lesbian employees. Journal of Applied Psychology, 86 (6), 1244-1261.

Randall, R., Griffiths, A., Cox, T., \& Welsh, C. (2002). The activation of mechanisms linking judgements of work design and management with musculoskeletal pain. Ergonomics, 45, 1331. 
Roessler, R. T., \& Rumrill, P. D. (1998). Reducing workplace barriers to enhance job satisfaction: An important post-employment service for employees with chronic illnesses. Journal of Vocational Rehabilitation, 10, 241-252.

Roth, P.L., \& BeVier, C.A. (1998). Response rates in HRM/OB survey research: norms and correlates, 1990-1994.

Sykes, M. A., Blanchard, E. B., Lackner, J., Keefer, L., \& Krasner, S. (2003).

Psychopathology in irritable bowel syndrome: Support for a psychophysiological model. Journal of Behavioral Medicine, 26 (4), 361-372.

Vickers, M. (1997). Life at work with 'invisible’ chronic illness (ICI): The 'unseen’, unspoken, unrecognised dilemma of disclosure. Journal of Workplace Learning, 7, 240-252.

VanEmmerik, H. (2002). Gender differences in the effects of coping assistance on the reduction of burnout in academic staff. Work \& Stress, 16 (3), 251-263.

Vivien, L. K. (2003). Managing HIV at the workplace: An empirical study of HIV and HR managers in Singapore. Journal of Occupational Health Psychology, 8, 235-246.

World Health Organization (1992). Health promotion and chronic diseases: Discovering a new quality of health. Copenhagen: World Health Organization Regional Office for Europe.

World Health Organization (1999). International Classification of Diseases - Version 10. Geneva: World Health Organization 